\title{
EDITORIAL
}

\section{Cannabis, cannabinoid receptors, and endocannabinoid system: yesterday, today, and tomorrow}

\begin{abstract}
Acta Pharmacologica Sinica (2019) 40:297-299; https://doi.org/ 10.1038/s41401-019-0210-3
\end{abstract}

Cannabis sativa, is also popularly known as marijuana, has been cultivated and used for recreational and medicinal purposes for many centuries. The main psychoactive content in cannabis is $\Delta^{9}$ tetrahydrocannabinol (THC). In addition to plant cannabis sativa, there are two classes of cannabinoids - the synthetic cannabinoids (e.g., WIN55212-2) and the endogenous cannabinoids (eCB), anandamide (ANA) and 2-arachidonoylglycerol (2-AG). The biological effects of cannabinoids are mainly mediated by two members of the G-protein-coupled receptor family, cannabinoid receptors $1\left(\mathrm{CB}_{1} \mathrm{R}\right)$ and $2\left(\mathrm{CB}_{2} \mathrm{R}\right)$. The endocannabinoids, cannabinoid receptors, and the enzymes/proteins responsible for their biosynthesis, degradation, and re-updating constitute the endocannabinoid system. In recent decades, the endocannabinoid system has attracted considerable attention as a potential therapeutic target in numerous physiological conditions, such as in energy balance, appetite stimulation, blood pressure, pain modulation, embryogenesis, nausea and vomiting control, memory, learning and immune response, as well as in pathological conditions such as Parkinson's disease, Huntington's disease, Alzheimer's disease, and multiple sclerosis.

$\mathrm{CB}_{1} \mathrm{R}$ is prominently expressed in the central nervous system (CNS) and has drawn great attention as it participates in a variety of brain function modulations, including executive, emotional, reward, and memory processing via direct interactions with the endocannabinoid system and indirect effects on the glutamatergic, GABAergic and dopaminergic systems. Unlike $\mathrm{CB}_{1} \mathrm{R}, \mathrm{CB}_{2} \mathrm{R}$ was considered as a "peripheral" cannabinoid receptor. However, this concept has been challenged recently by the identification of functional $C_{2}$ Rs throughout the central nervous system (CNS). When compared with $C B_{1} R$, brain $C B_{2} R$ exhibits several unique features: (1) $C B_{2}$ Rs have lower expression levels than $C B_{1} R s$ in the $C N S$, suggesting that $C B_{2} R s$ may not mediate the effects of cannabis under normal physiological conditions; (2) $\mathrm{CB}_{2} \mathrm{Rs}$ are dynamic and inducible; thus, under some pathological conditions (e.g., addiction, inflammation, anxiety, epilepsy etc.), $\mathrm{CB}_{2} \mathrm{R}$ expression can be upregulated in the brain, suggesting $C B_{2} R$ involvement in various psychiatric and neurological diseases; (3) brain $\mathrm{CB}_{2} \mathrm{Rs}$ are mainly expressed in neuronal somatodendritic areas (postsynaptic), while $C B_{1} R s$ are predominantly expressed in neuronal presynaptic terminals, suggesting an opposite role of $C_{1} R s$ and $C_{1} R s$ in regulation of neuronal firing and neurotransmitter release. Based on these characteristics, $C_{2}$ Rs have been considered to be an important substrate for neuroprotection, and targeting $C_{2} B_{2} R$ will likely offer a novel therapeutic strategy for treating neuropsychiatric and neurological diseases without $C B_{1} R$ mediated side effects.

The major goal of this Special Issue is to discuss and evaluate the current progress in cannabis and cannabinoid research in order to increase our understanding about cannabinoid action and the underlying biological mechanisms and promote the development cannabinoid-based pharmacotherapies.

In the article "Cannabidiol does not display drug abuse potential in mice behavior", the authors report original research to show that cannabidiol (CBD), the second major component in cannabis, lacks abuse potential [1]. This work will promote more CBD-related preclinical and clinical research in medication development for the treatment of neuropsychiatric diseases including substance use disorders. The article entitled "Computational systems pharmacology analysis of cannabidiol: a combination of chemogenomicsknowledgebase network analysis and integrated with silico modeling and simulation" systemically describes the potential targets of $\mathrm{CBD}$, the intracellular signaling pathways, and their associations with corresponding diseases. The authors used chemogenomics-knowledge base system and systematic network analysis of pharmacological action to generate CBD-target, targetpathway, and target-disease networks by combining both the results from the in silico analysis and the reported experimental validations [2]. The potential novel targets of CBD are discussed in "GPR3, GPR6, and GPR12 as novel molecular targets: their biological functions and interaction with cannabidiol", in which the authors show that CBD is an inverse agonist for the G-proteincoupled receptors 3, 6, and 12 (GPR3, GPR6, and GPR12) [3]. These orphan receptors may act as potential new molecular targets for $\mathrm{CBD}$ and therefore constitute new therapeutic targets for the treatment of various neurological and psychological diseases. In a review article "Brain activity of anandamide: a rewarding bliss?" the authors provide an overview from a preclinical perspective of the current state of knowledge regarding the behavioral pharmacology of ANA, with a particular emphasis on its motivational/reinforcing properties. They also discuss how modulation of ANA levels through inhibition of enzymatic metabolic pathways could provide a basis for developing new pharmacotherapeutic tools for the treatment of substance use disorders [4].

In this Special Issue, I have collected 7 articles that study and discuss cannabinoid receptors ( 3 for $C B_{1} R, 4$ for $C B_{2} R$ ). The research article "Cannabinoid $C_{1}$ receptor neutral antagonist AM4113 inhibits heroin self-administration without depressive side effects in rats" evaluates the potential utility of the $C B_{1} R$ neutral antagonist AM4113 in treatment of opioid abuse and addiction and compares the therapeutic anti-addictive effects and the possible side-effects of $A M 4113$ and the classical $C B_{1} R$ antagonist/inverse agonist SR141716A in animal models of addiction. The authors found that systemic administration of AM4113 significantly inhibited intravenous heroin selfadministration similar to SR141716A. However, SR141716A also exhibited aversive effect while AM4113 did not. These findings suggest that $A M 4113$ or other neutral $C B_{1} R$ antagonists may serve as a new class of $C_{1} B_{1} R$-based medications for the treatment of opioid addiction without SR141716A-like side-effects [5]. In the review article entitled "Translational potential of allosteric modulators targeting the cannabinoid $\mathrm{CB}_{1}$ receptor", the authors discuss recent advances in structural and mechanistic studies on $\mathrm{CB}_{1} \mathrm{R}$ allosteric modulators. They suggest that allosteric $\mathrm{CB}_{1}$ 
modulators provide tremendous opportunities to develop $\mathrm{CB}_{1}$ ligands with novel mechanisms of action; these ligands potentially improve the pharmacological effects and enhance drug safety in treating the disorders by regulating the functions of the $\mathrm{CB}_{1}$ receptor [6]. In a research article "Identification of novel mouse and rat $C B_{1} R$ isoforms and in silico modeling of human $C B_{1} R$ for peripheral cannabinoid therapeutics", the authors provide experimental evidence that human $\mathrm{CB}_{1} \mathrm{R}$ has a special isoform in peripheral but not in the CNS (called peripherally enriched $C_{1}$ isoforms), but this feature does not exist in rodents [7]. This study challenges the experimental approach that targeting peripheral $\mathrm{CB}_{1} \mathrm{R}$ is desirable for the treatment of metabolic syndromes without adverse neuropsychiatric effects using rodents.

For $\mathrm{CB}_{2} \mathrm{R}$ studies, in the article ${ }^{\prime} \mathrm{CB}_{2}$ receptor antibody signal specificity: correlations with the use of partial $\mathrm{CB}_{2}$-knockout mice and anti-rat $\mathrm{CB}_{2}$ receptor antibodies", authors address an important question in this research field regarding $\mathrm{CB}_{2} \mathrm{R}$ antibody signal specificity. Authors tested four commercial available $C_{2} R$ antibodies in wild-type and two strains of partial $C_{2} R$-knockout (KO) mice. The results suggest that none of the tested four polyclonal antibodies are highly mouse $\mathrm{CB}_{2} \mathrm{R}$-specific. Non-specific binding may be related to the expression of mutant or truncated $\mathrm{CB}_{2} \mathrm{R}$-like proteins in those partial $\mathrm{CB}_{2}-\mathrm{KO}$ mice and the use of antirat $C B_{2}$ antibodies because the epitopes are different between rat and mouse $\mathrm{CB}_{2} \mathrm{Rs}$ [8]. This work provides an important caution for using $C_{2} R$ antibodies to identify $C_{2} R$ expression in the future study. Xia et al. intent to answer another important question in cannabinoid research field, the heterogeneity. In the research article "Heterogeneity of cannabinoid ligand-induced modulations in intracellular $\mathrm{Ca}^{2+}$ signals of mouse pancreatic acinar cells in vitro" authors report a heterogenic effects of cannabinoid ligands on the intracellular $\mathrm{Ca}^{2+}$ signals in mouse pancreatic acinar cells, demonstrating that cannabinoid ligands exhibit the modulations of intracellular $\mathrm{Ca}^{2+}$ signals in a heterogenic manner through both cannabinoid receptors and non-receptor mechanisms [9]. In article "Protective effect of cannabinoid receptor 2specific agonist GW405833 on concanavalin A-induced acute liver injury", authors report that $\mathrm{CB}_{2} \mathrm{R}$ agonist GW405833 protects liver cells against acute concanavalin A-induced toxicity through the $\mathrm{CB}_{2} \mathrm{Rs}$ expressed in liver immune cells. The application of positron emission tomography (PET) in studying $C_{2} R$ function is summarized in a review article "Positron emission tomography (PET) of type 2 cannabinoid receptors for detecting inflammation in the central nervous system". Authors introduce several novels of $C_{2} R$ radiotracers that have been developed and evaluated to quantify microglial activation. Authors also summarize the recent preclinical and clinical imaging results of $\mathrm{CB}_{2} \mathrm{R}$ PET tracers and discuss the prospects of $\mathrm{CB}_{2} \mathrm{R}$ imaging using PET [10].

There are two review articles that discuss the endocannabinoid (eCB) system. The article "Integrating endocannabinoid signaling in the regulation of anxiety and depression" is focused on recent evidence that has added a new layer of complexity to the idea of targeting the eCB system for therapeutic benefits in neuropsychiatric diseases and on the future research direction of neural circuit modulations [11]. Another article "Endocannabinoid signaling in psychiatric disorders: a review of positron emission tomography studies" comprehensively reviewed PET studies examining differences in endocannabinoid signaling between individuals with psychiatric illness and healthy controls [12]. The possible cross-talk between the endocannabinoid and endovanilloid (vanilloid type 1, TRPV1) systems is discussed in the article "Developmental and behavioral effects in neonatal and adult mice following prenatal activation of endocannabinoid receptors by capsaicin". Here the authors found that prenatal exposure to capsaicin altered plus-maze performance, and the conditioned place preference/aversion paradigm following conditioning with capsaicin in adult animals [13]. Based on available data they suggest that the interaction between the endocannabinoid and endovanilloid signaling systems can be exploited for therapeutic applications in health and disease.

\section{PERSPECTIVES AND REMARKS}

Overall, the present special issue provides an overview and insight on pharmacological mechanisms and therapeutic potentials of cannabis, cannabinoid receptors, and eCB system. I believe that this special issue will promote further efforts to apply cannabinoid ligands as the therapeutic strategies for treating a variety of diseases. Although concepts and accumulating lines of evidence have been established, the challenges still remain in the cannabinoid research field and clinical practice. Following four aspects should be addressed in the future research regarding cannabis, cannabinoid receptors, and eCB system.

1. Heterogeneity: We should fully realize that cannabis, cannabinoid receptors, and $\mathrm{eCB}$ system exhibit high heterogeneity, from genes to receptors to intracellular G-protein-coupled signal pathways and membrane ion channels. For example, the same cannabinoid ligand (e.g., WIN55, 212-2) may produce biological responses through $C B$ receptor and non- $C B$ receptor mechanisms, and the same $C B$ receptors (e.g., $C B_{1} R$ ) may produce different biological effects.

2. Complexity: Cannabinoid system is very complex, including many ligands (plant-derived, synthetic and endogenous), various types of targets $\left(\mathrm{CB}_{1} \mathrm{R}, \mathrm{CB}_{2} \mathrm{R}, \mathrm{GRP} 55\right.$, TRPV1, and others), multi-signal pathways (ERK/MAPK and CAMP/PKA), and a lot of ion channels $\left(\mathrm{Ca}^{2+}, \mathrm{K}^{+}, \mathrm{Cl}^{-}\right.$and TRP channels).

3. Specificity: The most effort in cannabinoid research field is paid for identifying the specificity of cannabinoid effects. Since the above-mentioned heterogeneity and complexity, it is somehow difficult to distinguish cannabinoid's effects through a specific receptor $\left(C B_{1} R, C B_{2} R\right.$, or $C B_{1}-C B_{2}$ heterodimer), a specific signal pathway and a specific ion channel. Thus far, we still do not know the impact of this "specificity" in cannabinoid pharmacology and therapeutics. The key question, for instance, is which way is better for desired pharmacology and therapeutics: the specificity or multiple targets.

4. Inducible profile: Cannabinoid receptor, particularly brain $C_{2} R_{\text {, }}$ expression displays dynamic and inducible profiles under various pathological conditions. The inducible feature makes brain $\mathrm{CB}_{2} \mathrm{Rs}$ possible as therapeutic targets in the treatment of diseases without interruption of normal brain function.

\section{ADDITIONAL INFORMATION}

Competing interests: The authors declare no competing interests.

Jie $\mathrm{Wu}^{1,2}$

${ }^{1}$ Qingdao University Brain Science and Disease Institute, Qingdao 266021, China and ${ }^{2}$ St. Joseph's Hospital and Medical Center, Barrow Neurological Institute, Phoenix, AZ, USA Correspondence: Jie Wu (jie.wu@dignityhealth.org)

\section{REFERENCES}

1. Viudez-Martinez A, Garcia-Gutierrez MS, Medrano-Relinque J, Navarron CM, Navarrete F, Manzanares J. Cannabidiol does not display drug abuse potential in mice behavior. Acta Pharmacol Sin. 2019;40:358-64. https://doi.org/s41401-0180032-8.

2. Bian YM, He XB, Jing YK, Wang LR, Wang JM, Xie XQ. Computational systems pharmacology analysis of cannabidiol: a combination of chemogenomicsknowledgebase network analysis and integrated in silico modeling and simulation. Acta Pharmacol Sin. 2019;40:374-86. https://doi.org/s41401018-0071-1. 
3. Laun AS, Shrader SH, Brown KJ, Song ZH. GPR3. GPR6, and GPR12 as novel molecular targets: their biological functions and interaction with cannabidiol. Acta Pharmacol Sin. 2019;40:300-8. https://doi.org/s41401-018-0031-9.

4. Scherma M, Masia P, Satta V, Fratta W, Fadda P, Tanda G. Brain activity of anandamide: a rewarding bliss? Acta Pharmacol Sin. 2019;40:309-23. https://doi.org/ s41401-018-0075-x.

5. He XH, Jordan CJ, Vemuri K, Bi GH, Zhan J, Gardner EL, et al. Cannabinoid CB1 receptor neutral antagonist AM4113 inhibits heroin self-administration without depressive side effects in rats. Acta Pharmacol Sin. 2019;40:365-73. https://doi. org/s41401-018-0059-x.

6. Lu D, Immadi SS, Wu Z, Kendall DA. Translational potential of allosteric modulators targeting the cannabinoid CB1 receptor. Acta Pharmacol Sin. 2019;40:324-35. https://doi.org/s41401-018-0164-x.

7. Liu QR, Huang NS, Qu H, O'Connell JF, Gonzalez-Mariscal I, Santa-Cruz-Calvo S, et al. Identification of novel mouse and rat CB1R isoforms and in silico modeling of human CB1R for peripheral cannabinoid therapeutics. Acta Pharmacol Sin. 2019:40:387-97. https://doi.org/s41401-018-0152-1.

8. Zhang HY, Shen H, Jordan CJ, Liu QR, Gardner EL, Bonci A, et al. CB2 receptor antibody signal specificity: correlations with the use of partial $\mathrm{CB} 2$-knockout mice and anti-rat CB2 receptor antibodies. Acta Pharmacol Sin. 2019;40:398409. https://doi.org/s41401-018-0037-3.

9. Xia KK, Shen JX, Huang ZB, Song HM, Gao M, Chen DJ, et al. Heterogeneity of cannabinoid ligand-induced modulations in intracellular $\mathrm{Ca}^{2+}$ signals of mouse pancreatic acinar cells in vitro. Acta Pharmacol Sin. 2019;40:410-7. https://doi. org/s41401-018-0074-y.

10. Ni R, Mu L, Ametamey S. Positron emission tomography of type 2 cannabinoid receptors for detecting inflammation in the central nervous system. Acta Pharmacol Sin. 2019;40:351-7. https://doi.org/s41401-018-0035-5.

11. Yin $A Q$, Wang $F$, Zhang $X$. Integrating endocannabinoid signaling in the regulation of anxiety and depression. Acta Pharmacol Sin. 2019;40:336-41. https:// doi.org/s41401-018-0051-5.

12. Sloan ME, Grant CW, Gowin JL, Ramchandani VA, Le Foll B. Endocannabinoid signaling in psychiatric disorders: a review of positron emission tomography studies. Acta Pharmacol Sin. 2019;40:342-50. https://doi.org/s41401-018-0081-z.

13. Perchuk A, Bierbower SM, Canseco-Alba A, Mora Z, Tyrell L, Joshi N, et al. Developmental and behavioral effects in neonatal and adult mice following prenatal activation of endocannabinoid receptors by capsaicin. Acta Pharmacol Sin. 2019;40:418-24. https://doi.org/s41401-018-0073-z. 